\title{
LA GESTION \\ DES DÉCHETS RADIOACTIFS
}

I y a quatre ans, en 1988, le numéro de juillet-août de la RGN s'ornait en couverture d'un dessin représentant le futur centre de stockage de l'Aube, destiné aux déchets radioactifs à vie courte. Ce n'est plus un dessin mais une photo de ce même centre, aujourd'hui achevé, que nous publions à la «Une » du présent numéro. Ce raccourci symbolise le rythme à vrai dire assez rapide auquel "respirent " - tout au moins en France - les activités liées à la gestion de ces déchets... Au point que la RGN a résolu de présenter à ses lecteurs un nouveau dossier sur ce même sujet sans craindre, à quatre années d'intervalle, de quelconques redondances.

Les articles qu'on va lire décrivent des expériences, dégagent des enseignements, tracent des perspectives. Leur ambition est d'apporter sur ce sujet «sensible», point d'achoppement incontournable du débat nucléaire, l'information "transparente» que le député Christian Bataille appelle de ses vœux. Nous le remercions d'avoir bien voulu préfacer ce dossier et nous remercions les auteurs - qui sont aussi les acteurs directs des réalisations et recherches conduites dans ce domaine - d'avoir accepté de nous livrer la synthèse de leur expérience.

Nos remerciements vont également à $M$. Jacques Poirier dont le travail d'analyse et de coordination a été essentiel à l'élaboration de ce dossier.

Francis Sorin

\section{La gestion des déchets radioactifs : aperçu historique}

La gestion des déchets radioactifs a été l'objet depuis plus de quarante ans de l'attention des exploitants nucléaires et a constitué un thème de recherche identifié au CEA dès cette même époque.

Rappelons que les premiers verres radioactifs ont été élaborés à Saclay dès 1959, pour conduire à la mise en exploitation industrielle de l'AVM en 1978 et à celle de l'atelier de vitrification de La Hague (R7) en 1989. Ceci n'est qu'un exemple, certes spectaculaire, de continuité et de réussite technique mais les autres aspects de la gestion des déchets ont été également abordés et traités très tôt (le centre de stockage de la Manche a été mis en exploitation dès 1969).

Certes des développements technologiques et de nombreuses améliorations ou innovations ont été apportées aux techniques d'origine, conséquences normales des efforts de recherches consentis mais aussi rendus nécessaires par l'évolution des règles de sûreté.

Après les grandes étapes de réflexion des années 1980 (Groupe Castaing de 1981, 1983 et 1984, Groupe Goguel de 1985 à 1987, Groupe Permanent déchets créé en 1984, la définition des rôles respectifs des producteurs de déchets, du CEA organisme de recherches et de l'ANDRA), le début des années 1990 a été à la fois la concrétisation de grandes réalisations et l'époque de nouvelles grandes réflexions.

Dans les mises en exploitation industrielle citons celles de l'usine UP3 en août 1990 dont le fonctionnement met en évidence une diminution spectaculaire de certains déchets et une rationalisation du traitement des déchets effectué "en ligne". De même l'ouverture du centre de stockage de l'Aube en janvier 1992 est une concrétisation des souhaits du Groupe Castaing et des directives du ministère de 1984 .

Sur le plan réglementaire, sept règles fondamentales de sûreté ont été élaborées de 1982 à 1991.

Enfin, l'événement majeur est le vote de la loi sur les recherches visant à optimiser la gestion des déchets le 30 décembre 1991.

Tous ces points sont explicités dans les articles de la présente revue.

J. Lefèvre 